\title{
New Force-Free Models of Magnetic Clouds
}

\author{
M. Vandas
}

Astronomical Institute, Academy of Sciences, Bočni II 1401, 14131 Praha 4, Czech Republic

E. P. Romashets

Institute of Terrestrial Magnetism, Ionosphere, and Radio Wave Propagation, Russian Academy of Sciences, Troitsk, Moscow Region, 142190, Russia

\section{S. Watari}

Communications Research Laboratory, 4-2-1 Nukui-Kita, Koganei, Tokyo 184-8795, Japan

\begin{abstract}
Magnetic clouds are thought to be large flux ropes propagating through the heliosphere. Their twisted magnetic fields are mostly modeled by a constant-alpha force-free field in a circular cylindrical flux rope (the Lundquist solution). However, the interplanetary flux ropes are three dimensional objects. In reality they possibly have a curved shape and an oblate cross section. Recently we have found two force-free models of flux ropes which takes into account the mentioned features. These are (i) a constant-alpha force-free configuration in an elliptic flux rope (Vandas \& Romashets 2003, A\&A, 398, 801), and (ii) a nonconstant-alpha force-free field in a toroid with arbitrary aspect ratio (Romashets \& Vandas 2003, AIP Conf Ser. 679, 180). Two magnetic cloud observations were analyzed. The magnetic cloud of October 18-19, 1995 has been fitted by Lepping et al. (1997, JGR, 102, 14049) with use of the Lundquist solution. The cloud has a very flat magnetic field magnitude profile. We fitted it by the elliptic solution (i). The magnetic cloud of November $17-18,1975$ has been fitted by Marubashi (1997) with use of a toroidally adjusted Lundquist solution. The cloud has a large magnetic field vector rotation and a large magnetic field magnitude increase over the background level. We fitted it by the toroidal solution (ii). The both fits match the rotation of the magnetic field vector in a comparable quality to the former fits, but the description of the magnetic field magnitude profiles is remarkable better. It is possible to incorporate temporal effects (expansion) of magnetic clouds into the new solutions through a time-dependent alpha parameter as in Shimazu \& Vandas (2002, EP\&S, 54, 783).
\end{abstract}

This work was supported by grant 205/03/0953 from the Grant Agency of the Czech Republic, by project ME501 from MŠMT ČR, by project S1003006 from AV ČR, by EU/INTAS/ESA grant 99-00727, by CRDF grant TGP-944, and by the Japan Society for Promotion of Science. 